\title{
Multi-dimensional ambiguity function for subarray-based space-time coding radar
}

\author{
LAN Lan, LIAO Guisheng, XU Jingwei*, and WANG Hanbing
}

National Laboratory of Radar Signal Processing, Xidian University, Xi'an 710071, China

\begin{abstract}
Space-time coding radar has been recently proposed and investigated. It is a radar framework which can perform transmit beamforming at the receiver. However, the range resolution decreases when the number of the transmit element increases. A subarray-based space-time coding (sub-STC) radar is explored to alleviate the range resolution reduction. For the proposed radar configuration, an identical waveform is transmitted and it introduces a small time offset in different subarrays. The multidimensional ambiguity function of sub-STC radar is defined by considering resolutions in multiple domains including the range, Doppler, angle and probing direction. Analyses on properties of the multi-dimensional ambiguity function of the sub-STC radar with regard to the spatial coverage, resolution performance and low sidelobes are also given. Results reveal that the range resolution and low sidelobes performance are improved with the proposed approach.
\end{abstract}

Keywords: space-time coding radar, multi-dimensional ambiguity function, range resolution, wide spatial coverage, subarray, sidelobe.

DOI: $10.21629 /$ JSEE.2019.05.07

\section{Introduction}

Multiple-input and multiple-output (MIMO) radar has sparked intense interest from researchers over the past decade and been applied to various scenarios [1-3]. It is known that diverse waveforms are transmitted simultaneously from transmit antennas and received echoes are coherently processed in parallel at the receiver in the MIMO radar. Taking advantage of this waveform diversity, MIMO radar can obtain higher spatial resolutions and better detection performance. Besides, it provides extra degrees of freedom (DOFs) in the design of the radar systems and

\footnotetext{
Manuscript received March 01, 2018.

*Corresponding author.

This work was supported by the National Key Research and Development Program of China (2016YFE0200400), the Key R\&D Program of Shaanxi Province (2017KW-ZD-12), the Postdoctoral Science Foundation of Shaanxi Province, and the Nature Science Foundation of Shaanxi Province.
}

corresponding algorithms are developed [4]. Particularly, compared with the conventional phased-array radar, a greatly increased virtual aperture is obtained with the aid of orthogonal waveforms in MIMO radar [5]. However, the received signals are usually assumed to be orthogonal, but this is not a practical case for all Doppler and delay pairs [6]. Besides, MIMO radars gain the advantages of extra DOFs, however, there is absence of the transmit coherent processing gain [7].

The space-time coding (STC) radar which is also known as the coherent MIMO has been investigated in past several years [8,9]. As it benefits from a small time offset which is shifted in the transmitted signal, a wide angular coverage is achieved using coherent waveforms. And the ability of angular discrimination at the transmitter is maintained [10]. According to [11], a radar system with such a spacetime waveform transmission can significantly increase the agility for exploiting the capability of the transmitter in practice. In addition, by performing an angle-time twodimensional (2-D) matched filter, the equivalent transmit beamforming is achieved. Analyses on the performance of STC radar were presented in [9], where four different codes were addressed. In [12], authors provided an effective method to solve the problem of the "resonance" effect. The antenna coupling effects on the performance of a collocated coherent MIMO system was analyzed in [13] and a calibration technique was also presented. An original mismatched filtering approach based on a fixed spatial code was presented in [10] which can optimize the sidelobes of the ambiguity function.

The ambiguity function for radar signals, which is determined by the matched filter, was firstly introduced by Woodward [14]. Since the 60s, researchers has initiated the study of the multi-dimensional ambiguity function in linear arrays. It was firstly studied in a surveillance scenario where a receiver with different delay sets was used to form multiple beams for different directions [15]. In the case of single-input-single-output (SISO) radar systems, 
the definition of the ambiguity function is used to evaluate the distortion of the received signal which is matched to a specific transmitted signal with diverse time delays and Doppler shifts [16,17]. Nevertheless, in MIMO radars, the system geometry and the orthogonality of different waveforms are vital parameters which should be considered in evaluating the performance of MIMO radar systems. The properties of the ambiguity function for different radar frameworks including the MIMO radar [18,19] and the phased-MIMO radar [20] are investigated. Methods to select system parameters can be also found in [20]. For STC array radar, the ambiguity function, concerning each probing direction and range, has been illustrated in the presented works. However, the analysis about the Doppler property remains to be investigated.

It is necessary to investigate the ambiguity function and to analyze the angle, velocity, and range resolutions. More specifically, it is known that the angle resolution, the velocity resolution (also termed as the Doppler resolution) as well as the range resolution depend on the spatial aperture of the antenna, the signal duration in time, and the bandwidth of the transmitted signal, respectively [14]. The range and velocity resolutions are major tools for examining the waveform of a radar system. And a radar system with multiple waveforms results in a wide beam, which is an effective solution to improve the angle and velocity resolutions for detecting targets in a complex environment [21]. However, the improvement of the velocity resolution is obtained at the cost of the non-directive beam at the transmitter, which results in a poorer rejection for echoes from adjacent directions [22]. To solve this problem, the STC radar provides a feasible solution to recover the angular separation at the transmitter. Hence, the signals transmitted in different directions are separable at the receiver. Two approaches to improve both the angle and velocity resolutions and to reject the interferences were proposed in [20]. Nevertheless, the range resolution degrades with the increment of the transmit element number. Besides, the angle resolution also degrades with such space-time codes [20].

In this paper, the configuration of the subarray-based space-time coding (sub-STC) radar is presented and its multi-dimensional ambiguity function is studied. The subSTC radar is characterized by shifting an identical waveform with a small time offset across adjacent subarrays which can be either regular or irregular. Hence, the definition of the ambiguity function is extended to the multidimensional case where resolutions in multiple domains including the range, Doppler and angle are considered. And analyses on the properties of the multi-dimensional ambiguity function in sub-STC radar with regard to the spatial coverage, the resolution performance and the low sidelobes are given. By comparing with the conventional STC radar, the sub-STC radar benefits from these characteristics. And deep insights into radar configurations are provided. With the proposed sub-STC radar, the range resolution is enhanced and the DOF is increased. Moreover, it reduces sidelobes of the ambiguity function.

The remainder of this paper is organized as follows. Section 2 investigates the signal model of the sub-STC radar systems. The multi-dimensional ambiguity function of the sub-STC radar including parameters of angle, probing direction, range and Doppler is analyzed in Section 3. Simulation results are shown in Section 4. Section 5 concludes the whole paper with future work.

\section{Signal model of the sub-STC radar}

For the sake of clarity and without loss of generality, assume there is a uniform linear array with $M$ halfwavelength spaced array elements. It is assumed that the array elements are used for both transmission and reception. It is shown in Fig. 1 that the array is divided into $K$ subarrays and the number of elements in the $k$ th subarray is $M_{k}\left(\sum_{k=1}^{K} M_{k}=M, M_{k} \leqslant M\right)$.
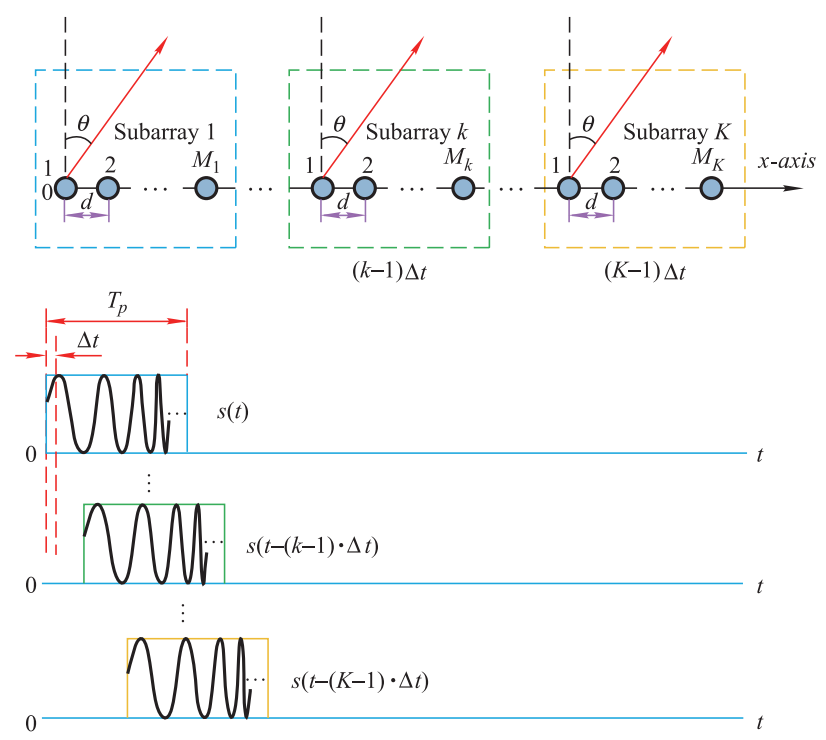

Fig. 1 Configuration of the sub-STC radar

We consider both the regular and irregular arrays where the number of elements are identical and different within each subarray, respectively. Then, a waveform $\varphi(t)$ within each subarray is coherently transmitted while the waveform is shifted by a small time offset in different subarrays. The baseband waveform transmitted at the $k$ th subarray is written as

$$
\varphi_{k}(t)=\varphi(t-(k-1) \Delta t), \quad k=1, \ldots, K
$$


where $\Delta t=1 / B$ refers to the time offset in sub-STC radar, and $B$ denotes the bandwidth of the system. The $\Delta t$ is small compared with the pulse width, and it has the relationship with the time-bandwidth (BT) product, i.e., $\mathrm{BT}=T_{p} / \Delta t$. Notably, the BT product should be large in a sub-STC system.

In general, the amplitude of the transmitted waveform is assumed to be 1 . In sub-STC radar, $\varphi_{k}(t)$ is identical within the $k$ th subarray, and the transmitted waveform of the $k$ th subarray is

$$
s_{k}(t)=\varphi(t-(k-1) \Delta t) \exp \left\{\mathrm{j} 2 \pi f_{0} t\right\}
$$

where $f_{0}$ is the carrier frequency.

The frequency-time representation and the spectra of the sub-STC radar with linear frequency modulation (LFM) waveforms are respectively shown in Fig. 2.

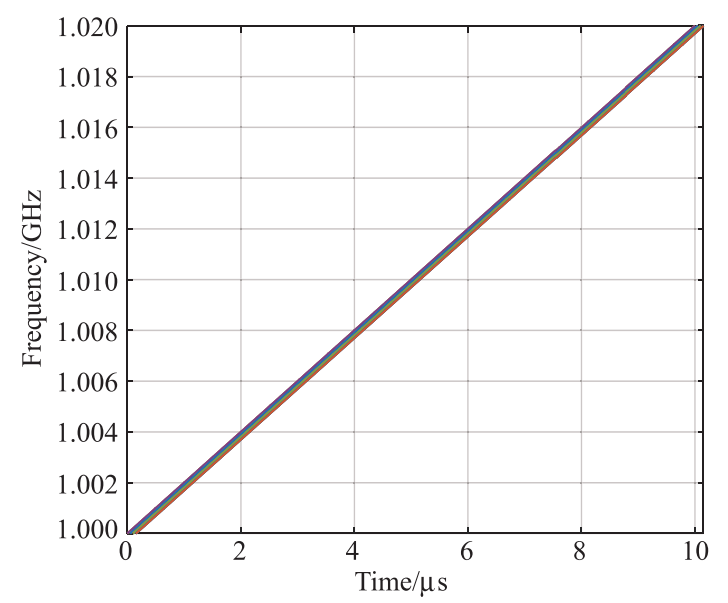

(a) Frequency-time representation

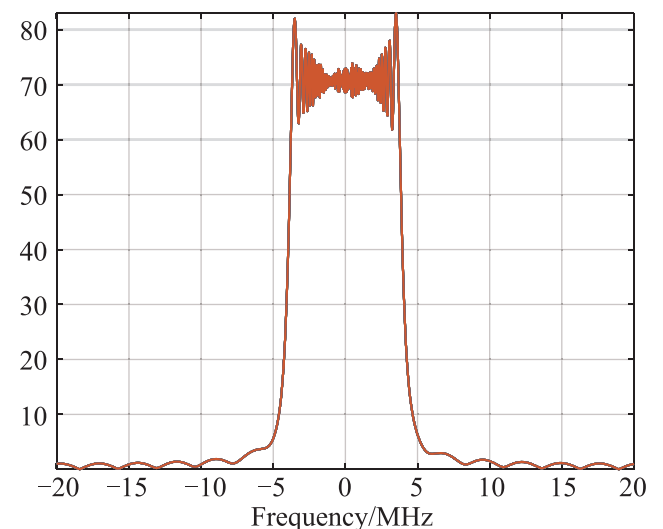

(b) The spectra

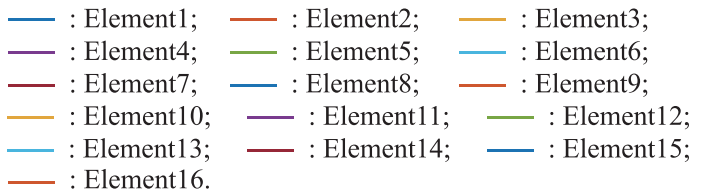

Fig. 2 Sub-STC radar with $\mathrm{LFM}$ waveforms, $M=16, K=4$
It can be seen from Fig. 2(a) that the waveform is identical in each subarray but it shifts with $\Delta t$ across diverse subarrays, while the transmitted waveforms occupy the same band in the frequency domain. In comparison with the frequency diverse array (FDA), carrier frequencies are linearly increased across array elements [23-25] in FDA but the frequencies are identical in sub-STC radar.

It follows from (2) that, the phase difference of the chirp between two adjacent subarrays for a specific instant is expressed as

$$
\Delta \phi=2 \pi f \cdot \Delta t=2 \pi \cdot \frac{f}{B}
$$

where $f \in\left[f_{0}-\frac{B}{2}, f_{0}+\frac{B}{2}\right]$. Thus, $\Delta \phi \in\left[\phi_{0}-\pi, \phi_{0}+\right.$ $\pi]$ with $\phi_{0}=2 \pi \frac{f_{0}}{B}$. Notably, the phase shift changes with $2 \pi$ within the bandwidth. Therefore, the coverage of the full angular space is achieved. Actually, different frequencies are corresponding to different spatial angles, namely, the wide spatial coverage is obtained at the cost of a large bandwidth.

Assume that there is a stationary target which locates in far-field at angle $\theta$ and range $R$. Considering the narrowband assumption, the signal of the $m_{k}$ th $\left(m_{k}=\right.$ $1,2, \ldots, M_{k}$ ) element in the $k$ th subarray reaching the target is

$$
\begin{gathered}
s_{k}\left(t-\tau_{m}\right)=\varphi\left(t-(k-1) \Delta t-\tau_{m}\right) . \\
\exp \left\{\mathrm{j} 2 \pi f_{0}\left(t-\tau_{m}\right)\right\} \approx \varphi\left(t-(k-1) \Delta t-\frac{\tau_{0}}{2}\right) . \\
\exp \left\{\mathrm{j} 2 \pi f_{0}\left(t-\tau_{m}\right)\right\}=s_{k}\left(t-\frac{\tau_{0}}{2}\right) . \\
\exp \left\{\mathrm{j} \frac{2 \pi}{\lambda}\left(\sum_{i=0}^{k-1} M_{i}+m_{k}-1\right) d \sin \theta\right\}
\end{gathered}
$$

where $\tau_{m}=\frac{1}{c}\left(R-d\left(\sum_{i=0}^{k-1} M_{i}+m_{k}-1\right) \sin \theta\right)$ denotes the $m_{k}$ th time delay of the element in the $k$ th subarray, $\varphi\left(t-\tau_{m}\right) \approx \varphi\left(t-\frac{\tau_{0}}{2}\right), \tau_{0}=\frac{2 R}{c}, \lambda=\frac{c}{f_{0}}$ is the wavelength, $c$ denotes the speed of the light, $d=\frac{\lambda}{2}$ refers to the interval between adjacent elements, and $\theta$ is the angle of view. In this paper, we adopt the LFM signal.

More specifically, the signals which are transmitted by all elements within the $k$ th subarray can be written as

$$
\begin{aligned}
s_{\mathrm{T} k}(t, \theta)= & s_{k}\left(t-\frac{\tau_{0}}{2}\right) \cdot \exp \left\{\mathrm{j} \frac{2 \pi}{\lambda}\left(\sum_{i=0}^{k-1} M_{i}\right) d \sin \theta\right\} . \\
& \sum_{m_{k}=1}^{M_{k}} \exp \left\{\mathrm{j} \frac{2 \pi d}{\lambda}\left(m_{k}-1\right) \sin \theta\right\}
\end{aligned}
$$

where $M_{0}=0$. It can be seen that $s_{\mathrm{T} k}(t, \theta)$ is angle-timedependent in sub-STC radar. 
Subsequently, the overall signal collected from $K$ subarrays is

$$
\begin{gathered}
s_{\mathrm{T}}(t, \theta)=\sum_{k=1}^{K} s_{\mathrm{T} k}(t, \theta)= \\
\sum_{k=1}^{K}\left[s_{k}(t) \cdot \exp \left\{\mathrm{j} \frac{2 \pi}{\lambda}\left(\sum_{i=0}^{k-1} M_{i}\right) d \sin \theta\right\} .\right. \\
\left.\sum_{m_{k}=1}^{M_{k}} \exp \left\{\mathrm{j} \frac{2 \pi}{\lambda}\left(m_{k}-1\right) d \sin \theta\right\}\right] .
\end{gathered}
$$

Considering the round-time propagation, the signal which is transmitted by the $m_{k}$ th element of the $k$ th subarray and received by the $n$th $(n=1,2, \ldots, M)$ element is

$$
\begin{gathered}
s_{m, n}\left(t-\tau_{0}, \theta\right)=\beta s_{k}\left(t-\tau_{0}\right) . \\
\exp \left\{j \frac{2 \pi}{\lambda}\left(\sum_{i=0}^{k-1} M_{i}+m_{k}-1\right) d \sin \theta\right\} . \\
\exp \left\{j \frac{2 \pi}{\lambda}(n-1) d \sin \theta\right\}
\end{gathered}
$$

where $\beta$ denotes the coefficient of the far-field point source.

The whole echoes received by the $n$th element is expressed as

$$
\begin{gathered}
s_{R}^{n}(t, \theta)=\sum_{k=1}^{K} s_{m, n}\left(t-\tau_{0}, \theta\right)= \\
\beta \cdot \exp \left\{\mathrm{j} \frac{2 \pi d}{\lambda}(n-1) \sin \theta\right\} \sum_{k=1}^{K} s_{k}\left(t-\tau_{0}\right) . \\
\sum_{m_{k}=1}^{M_{k}} \exp \left\{\mathrm{j} \frac{2 \pi}{\lambda}\left(\sum_{i=0}^{k-1} M_{i}+m_{k}-1\right) d \sin \theta\right\} .
\end{gathered}
$$

In the process, an angle-time 2-D matched filter is devised to process the received echoes for a possible specific direction $\theta_{0}$ and the formulation of the angle-time 2-D matched filter in the sub-STC radar can be expressed as

$$
\begin{gathered}
h\left(t, \theta_{0}\right)=\sum_{k=1}^{K}[\varphi(t-(k-1) \Delta t) . \\
\left.\sum_{m_{k}=1}^{M_{k}} \exp \left\{\mathrm{j} \frac{2 \pi}{\lambda}\left(\sum_{i=0}^{k-1} M_{i}+m_{k}-1\right) d \sin \theta_{0}\right\}\right] .
\end{gathered}
$$

Hence, the equivalent transmit beamforming is performed at the receiver. The matched filter is utilized to maximize the output signal-to-noise ratio (SNR). And the output result of the $n$th receiver channel at $\theta_{0}$ can be calculated as

$$
y_{n}\left(t, \theta_{0}\right)=s_{R}^{n}(t, \theta) * h^{*}\left(t, \theta_{0}\right)
$$

where $[\cdot]^{*}$ denotes the complex conjugate operator and $*$ denotes the convolution operator.

\section{Multi-dimensional ambiguity function of sub-STC radar}

In traditional radar systems, the ambiguity function is utilized to characterize the range and Doppler resolutions of a radar system. It is a major tool to mathematically analyze the properties of a specific radar waveform. Recall that in MIMO systems, the received signal in each receiver channel consists of a sum of transmitted signals. And a bank of matched filters are constructed where each of these filters has a kernel function given by one of the transmitted waveforms. Hence, the ambiguity function of the MIMO radar can be regarded as the inner product of received echoes under two sets of target parameters, which can be used to evaluate the performance of the transmitted signals without noise. As the complex output of a linear antenna array in the sub-STC radar is a function of angle and time, the concept of the multi-dimensional ambiguity function is extended to sub-STC by considering angle, each probing direction, range and Doppler shift. It also means that the radar waveform affects resolutions in the range, Doppler as well as the spatial angle domains.

$$
\begin{gathered}
\chi\left(\tau, \theta, \theta_{0}, f_{d}\right)=\left|\int_{-\infty}^{\infty} h\left(t, \theta_{0}\right) \cdot h^{*}(t-\tau, \theta) \mathrm{e}^{\mathrm{j} 2 \pi f_{d} t} \mathrm{~d} t\right|= \\
\mid \int_{-\infty}^{\infty} \sum_{k=1}^{K}\left[\varphi(t-(k-1) \Delta t) \cdot \sum_{m_{k}=1}^{M_{k}} \exp \left\{\mathrm{j} \frac{2 \pi}{\lambda}\left(\sum_{i=0}^{k-1} M_{i}+m_{k}-1\right) d \sin \theta_{0}\right\}\right] . \\
\sum_{q=1}^{K}\left[(\varphi(t-(q-1) \Delta t-\tau))^{*} \cdot \sum_{m_{q}=1}^{M_{q}} \exp \left\{-\mathrm{j} \frac{2 \pi}{\lambda}\left(\sum_{i=0}^{q-1} M_{i}+m_{q}-1\right) d \sin \theta\right\}\right] \mathrm{e}^{\mathrm{j} 2 \pi f_{d} t} \mathrm{~d} t \mid \\
\bar{\chi}_{\theta_{\theta_{0}=0^{\circ}}}(\tau, \theta)=\mid \int_{-\infty}^{\infty} \sum_{k=1}^{K}[\varphi(t-(k-1) \Delta t)] \cdot \sum_{q=1}^{K}\left[(\varphi(t-(q-1) \Delta t-\tau))^{*} .\right.
\end{gathered}
$$




$$
\begin{gathered}
\left.\sum_{m_{q}=1}^{M_{q}} \exp \left\{-\mathrm{j} \frac{2 \pi}{\lambda}\left(\sum_{i=0}^{q-1} M_{i}+m_{q}-1\right) \cdot d \sin \theta\right\}\right] \mathrm{d} t \mid \\
\bar{\chi}_{\substack{\tau=0 \\
f_{d}=0}}\left(\theta, \theta_{0}\right)=\mid \int_{-\infty}^{\infty} \sum_{k=1}^{K}\left[\varphi(t-(k-1) \Delta t) \sum_{m_{k}=1}^{M_{k}} \exp \left\{\mathrm{j} \frac{2 \pi}{\lambda}\left(\sum_{i=0}^{k-1} M_{i}+m_{k}-1\right) d \sin \theta_{0}\right\}\right] . \\
\sum_{q=1}^{K}\left[(\varphi(t-(q-1) \Delta t))^{*} \sum_{m_{q}=1}^{M_{q}} \exp \left\{-\mathrm{j} \frac{2 \pi}{\lambda}\left(\sum_{i=0}^{q-1} M_{i}+m_{q}-1\right) d \sin \theta\right\}\right] \mathrm{d} t \mid \\
\bar{\chi}_{\theta=\theta_{0}}\left(\tau, f_{d}\right)=\left|\int_{-\infty}^{\infty} \sum_{k=1}^{K}[\varphi(t-(k-1) \Delta t)] \cdot \sum_{q=1}^{K}\left[(\varphi(t-(q-1) \Delta t-\tau))^{*}\right] \cdot \mathrm{e}^{\mathrm{j} 2 \pi f_{d} t} \mathrm{~d} t\right| \\
\bar{\chi}_{\theta_{0}=0^{\circ}}\left(\theta, f_{d}\right)=\mid \int_{-\infty}^{\infty} \sum_{k=1}^{K}[\varphi(t-(k-1) \Delta t)] \cdot \sum_{q=1}^{K}\left[(\varphi(t-(q-1) \Delta t))^{*} .\right. \\
\left.\sum_{m_{q}=1}^{M_{q}} \exp \left\{-\mathrm{j} \frac{2 \pi}{\lambda}\left(\sum_{i=0}^{q-1} M_{i}+m_{q}-1\right) d \sin \theta\right\}\right] \cdot \mathrm{e}^{\mathrm{j} 2 \pi f_{d} t} \mathrm{~d} t \mid \cdot
\end{gathered}
$$

For the sub-STC radar, the ambiguity function can be defined in (11), where $|\cdot|$ represents the envelope detector, $\tau$ is time delay, $f_{d}$ is Doppler shift, $\varphi(t)=\operatorname{rect}\left(\frac{t}{T_{p}}\right) \exp \left\{\mathrm{j} \pi \frac{B}{T_{p}} t^{2}\right\}$, and $\operatorname{rect}\left(\frac{t}{T_{p}}\right)=$ $\left\{\begin{array}{ll}1, & |t| \leqslant T_{p} \\ 0, & \text { otherwise }\end{array}\right.$ denotes an impulse function.

As is shown in (11), the multi-dimensional ambiguity function $\chi\left(\tau, \theta, \theta_{0}, f_{d}\right)$ consists of four parameters, i.e., angle $\theta$, each probing direction $\theta_{0}$, range $R=c \cdot \tau / 2$ and Doppler phase $f_{d}$. The first bracket is referred to as the signal transmitted in angle $\theta_{0}$, which is similar to the transmit beampattern with the static weight vector pointing at $\theta_{0}$. The second bracket denotes the replica of the expected signal with direction $\theta$ and time delay $\tau$.

It is noteworthy that when $K=1$, the whole transmit array is transformed to the conventional array without subarrays, emitting an identical waveform $\varphi(t)$, then the signal model simplifies to the conventional signal model, i.e., the phased-array radar. Moreover, when $K=M$, the signal model transfers to the STC radar.

To determine the performance of the multi-dimensional ambiguity function of the sub-STC radar, the multidimensional ambiguity function can be divided into four 2-D slices: $\bar{\chi}_{\substack{f_{d}=0 \\ \theta_{0}=0^{\circ}}}(\tau, \theta)$ in (12) is the range-angle 2-D slice which denotes the relationship of the range and angle at a fixed direction; the angle-angle 2-D slice $\bar{\chi}_{\substack{\tau=0 \\ f_{d}=0}}\left(\theta, \theta_{0}\right)$ in (13) is a function of both the $\theta_{0}$ and $\theta$, which evaluates the transmit spatial angular coverage of the target; the
range-Doppler 2-D slice $\bar{\chi}_{\theta=\theta_{0}}\left(\tau, f_{d}\right)$ in (14) is the radar ambiguity diagram, which is used to explore the range resolution and the Doppler resolution; the angle-Doppler 2-D slice $\bar{\chi}_{\theta_{0}=0^{\circ}}\left(\theta, f_{d}\right)$ in (15) describes the angleDoppler relationship for sub-STC radar with LFM waveforms.

The STC radar shifts an identical waveform with a small time offset through the transmit antenna array elements. With this radar framework, the orthogonality of the radiated signals can be acquired since the STC radar can be regarded as the coherent MIMO radar [11]. In an STC radar system, multiple equivalent transmit beams are simultaneously formed at the receiver [9]. Hence, it simultaneously achieves the high angular resolution and instant wide angular coverage, and the low-probability of interception can be realized in the STC radar. The sub-STC maintains the advantages of the conventional STC radar, in the meanwhile, more properties can be obtained. Analyses on the performance of the multi-dimensional ambiguity function in sub-STC radar are given in the following aspects:

(i) Spatial coverage

As no amplitude variation can be found along the main diagonal in the angle-angle 2-D cut of the ambiguity function in the STC radar, it reveals the fact that for all observed spatial angles, the level of the transmitted signal is identical [11]. In other words, the whole transmitted energy can be equally distributed along the spatial angle space [20]. Although the wide spatial coverage is obtained, the resolution degrades.

(ii) Resolution

In the STC radar, the range resolution varies with the 
change of the transmit array elements number. In particular, only the fraction $\frac{1}{M}$ of the bandwidth is sent for one specific angle [20], or other, the resolution is related with the bandwidth and the element number. It means that the range resolution decreases with the growth of the element number. Although the combination of the STC radar with phase-coded waveforms can help with the improvement of the range resolution, however, it leads to high range sidelobes.

\section{(iii) Low sidelobes}

The low range sidelobes are due to the fact that a weight in the space and time domain is added. Nevertheless, the sidelobe peak is still high in the angle domain of the multidimensional ambiguity function which may result in failure detection of targets. The high sidelobes can be mitigated with a suitable windowing operation in the time domain. Various classic windows have been used to reduce the sidelobes [26]. Although sidelobes are reduced with windowing, the width of the mainlobe increases and the processing gain is low, which is not a satisfying result. Moreover, optimization methods including the genetic algorithm [27], artificial bee colony [28], particle swarm optimization [29] are applied. However, the system may suffer from high complexity, which is not desired in practice.

The combination of the subarray-based method with the STC radar shares the characteristics of both the STC and the phased-MIMO radar. Therefore, the sub-STC radar framework can cope with these problems, which will be provided in details in the next section.

\section{Simulation results}

Numerical simulation examples are carried out to corroborate the effectiveness and merits of the proposed sub-STC radar in this section. Parameters are given in Table 1.

Table 1 System parameters

\begin{tabular}{cc}
\hline Parameter & Value \\
\hline Element number $M$ & 16 \\
Subarray number $K$ & 4 \\
Carrier frequency $f_{0} / \mathrm{GHz}$ & 1 \\
Wavelength $\lambda / \mathrm{m}$ & 0.3 \\
Bandwidth $B / \mathrm{MHz}$ & 20 \\
Pulse repetition frequency/kHz & 20 \\
Time shift $\Delta t / \mu \mathrm{s}$ & 0.05 \\
Sampling frequency $/ \mathrm{MHz}$ & 100 \\
BT product & 200 \\
\hline
\end{tabular}

Fig. 3 presents the multi-dimensional ambiguity function in the STC radar where the number of transmit elements is $M=16$. The angle-angle 2-D cut displays that in the STC radar, the radiation for all spatial angles is uniform. Thanks to the advantage of wide spatial coverage, the target can be found at any angle. However, the mainlobe width in the range domain is wide, which means a range resolution reduction. Besides, in practice, it is required that the sidelobes should be low, and it is supposed to reduce the sidelobes of the multi-dimensional ambiguity function.

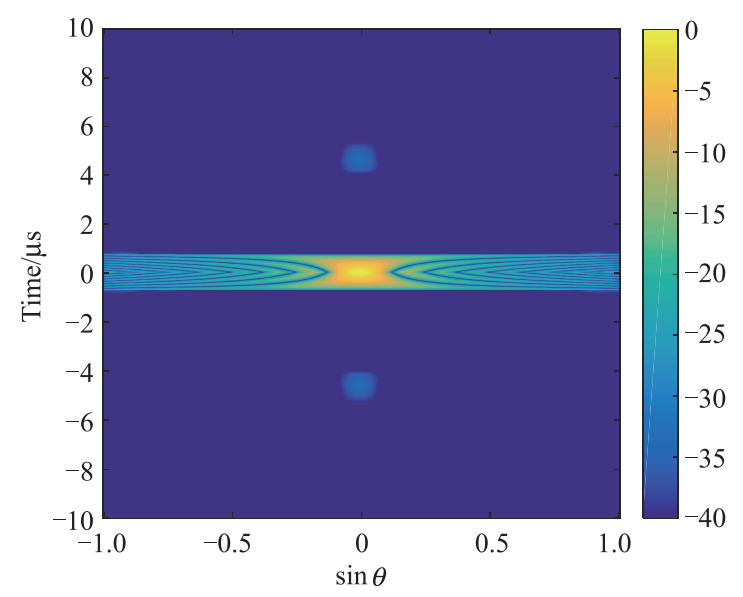

(a) Range-angle 2-D slice

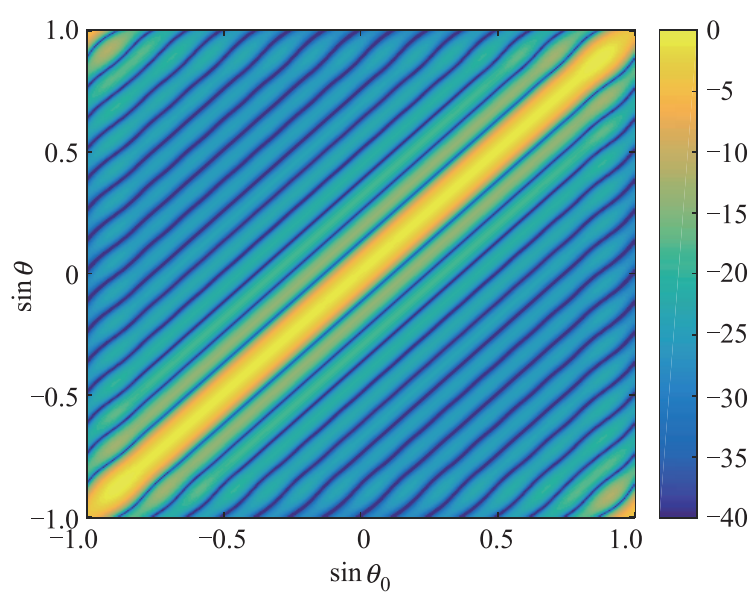

(b) Angle-angle 2-D slice

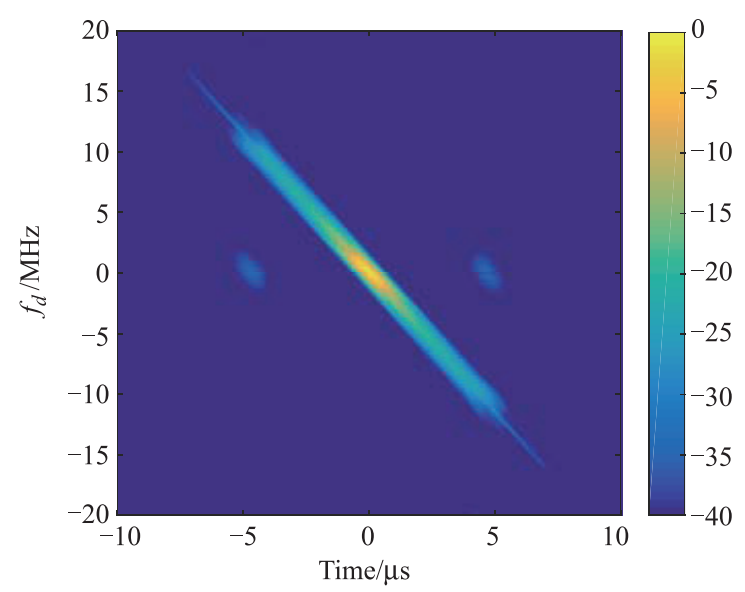

(c) Range-Doppler 2-D slice 


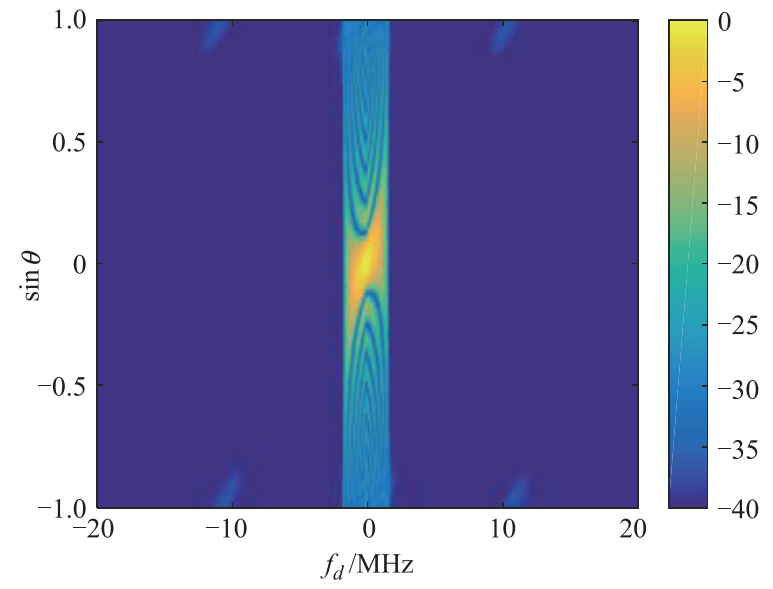

(d) Angle-Doppler 2-D slice

Fig. 3 Different 2-D slices of multi-dimensional ambiguity function in STC radar

Fig. 4 -Fig. 7 display the multi-dimensional ambiguity function of the proposed sub-STC radar.

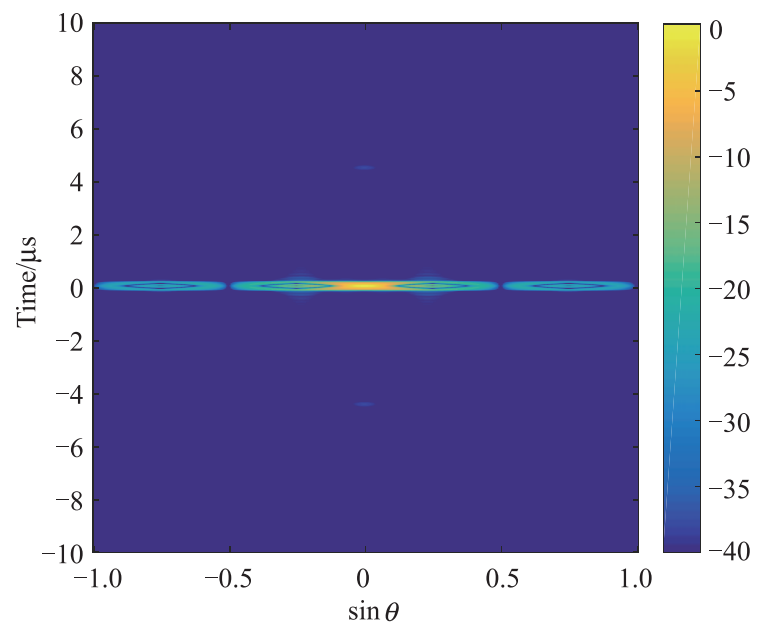

(a) Range-angle 2-D slice with regular subarrays

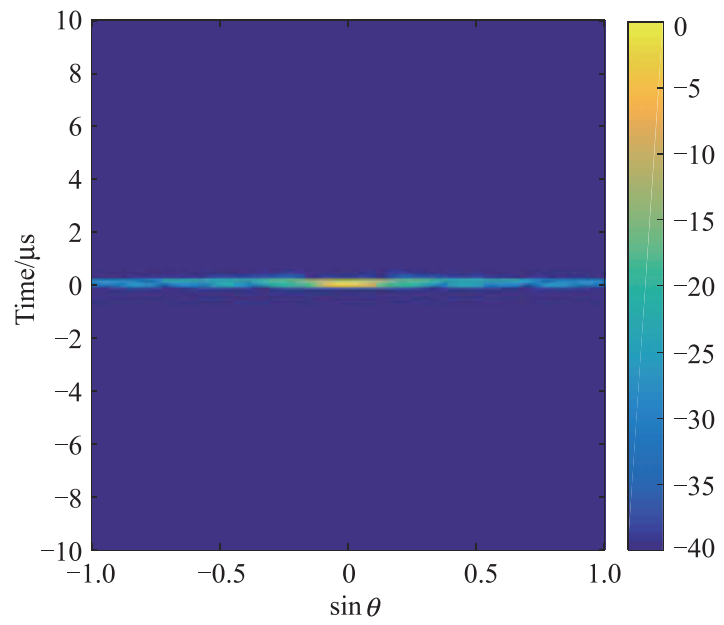

(b) Range-angle 2-D slice with irregular subarrays

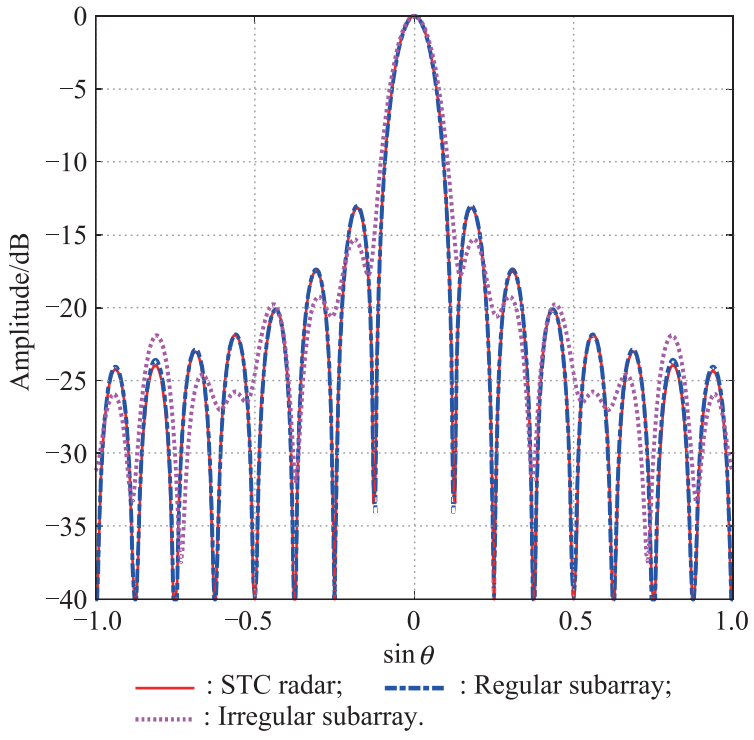

(c) Angle-dependent plot at $\tau=0$

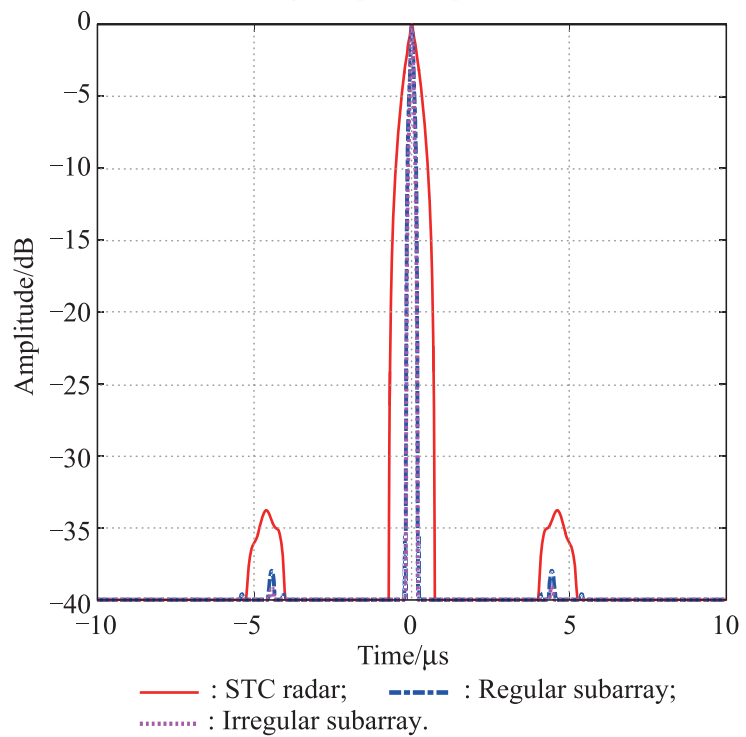

(d) Range-dependent plot at $\theta_{0}=0^{\circ}$

Fig. 4 Range-angle 2-D slices of sub-STC radar

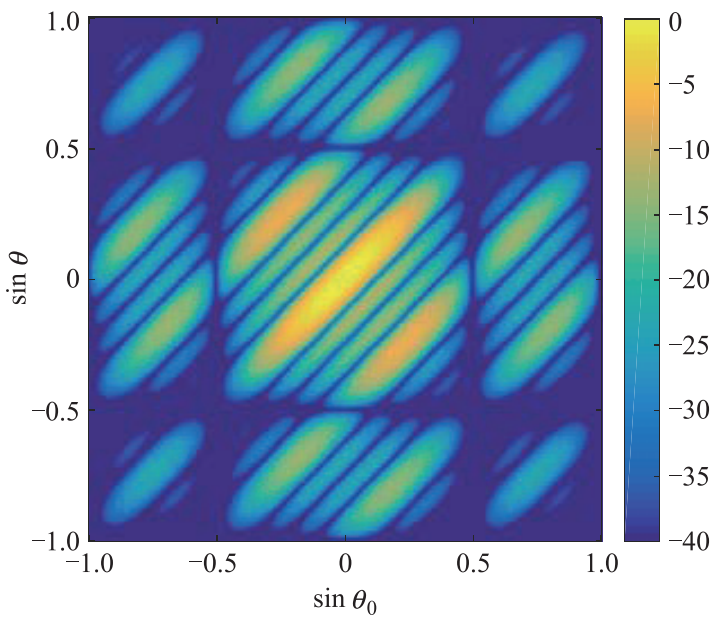

(a) Angle-angle 2-D slice with regular subarrays 


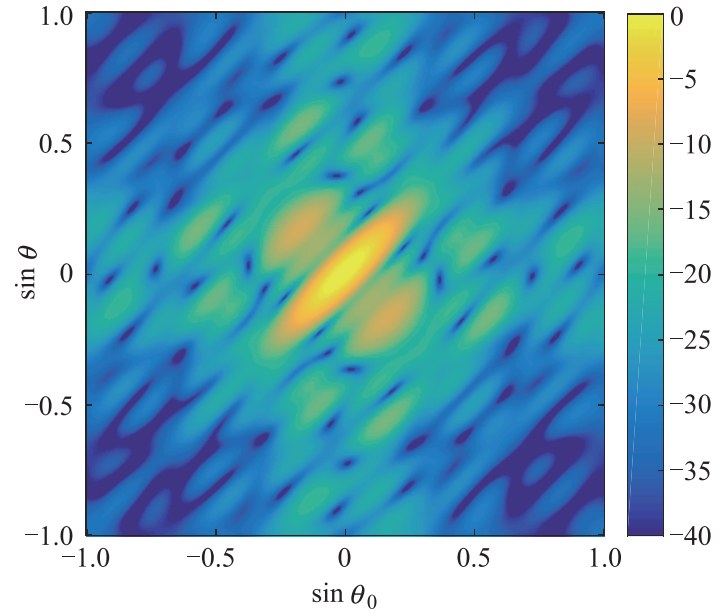

(b) Angle-angle 2-D slice with irregular subarrays

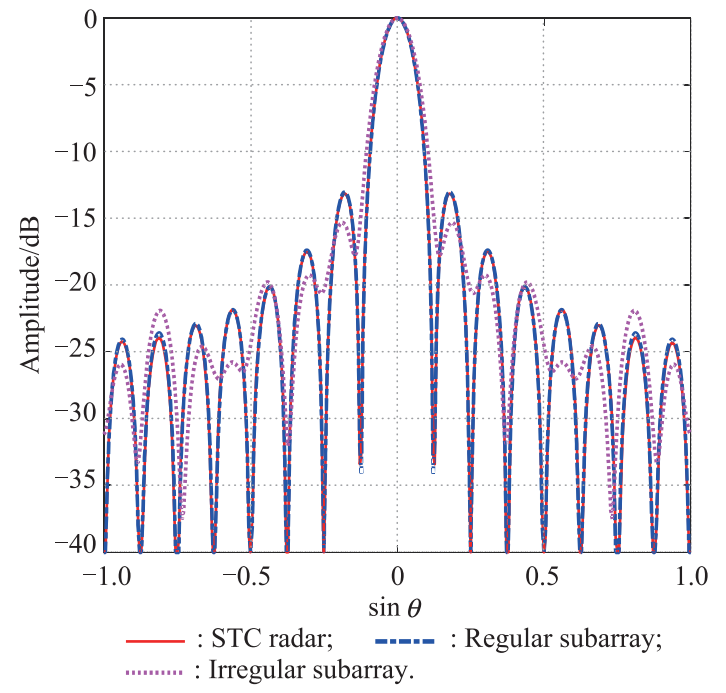

(c) Angle-dependent plot at $\theta=0^{\circ}$

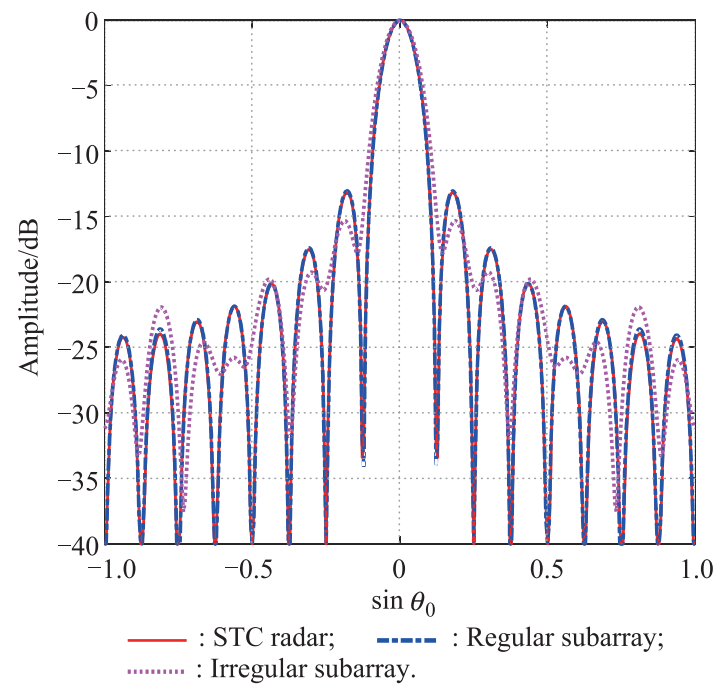

(d) Angle-dependent plot at $\theta=0^{\circ}$

Fig. 5 Angle-angle 2-D slices of sub-STC radar $M_{1}=\cdots=M_{4}=4$ is assumed for regular subarrays, and for irregular subarrays, assume $M_{1}=2, M_{2}=3$, $M_{3}=5$, and $M_{4}=6$. To investigate the performance of the proposed method, simulation results about the low sidelobes performance, the spatial coverage and the resolution are discussed.

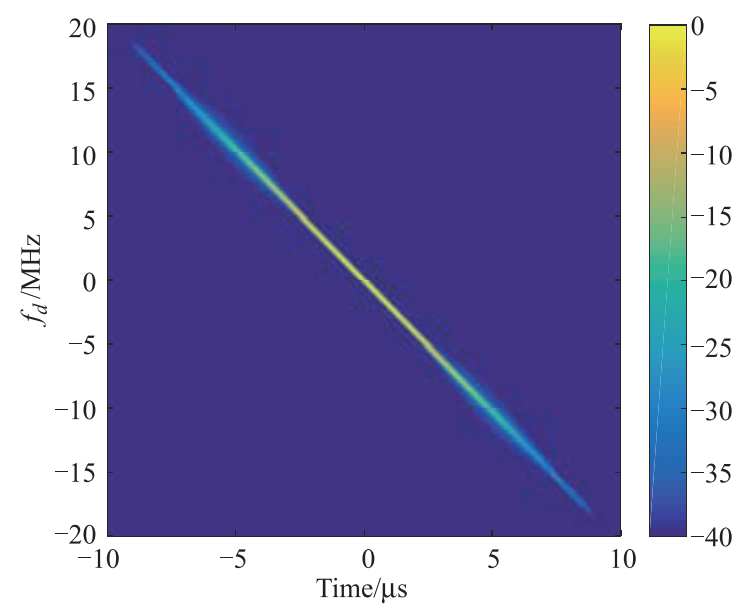

(a) Range-Doppler slice with regular subarrays

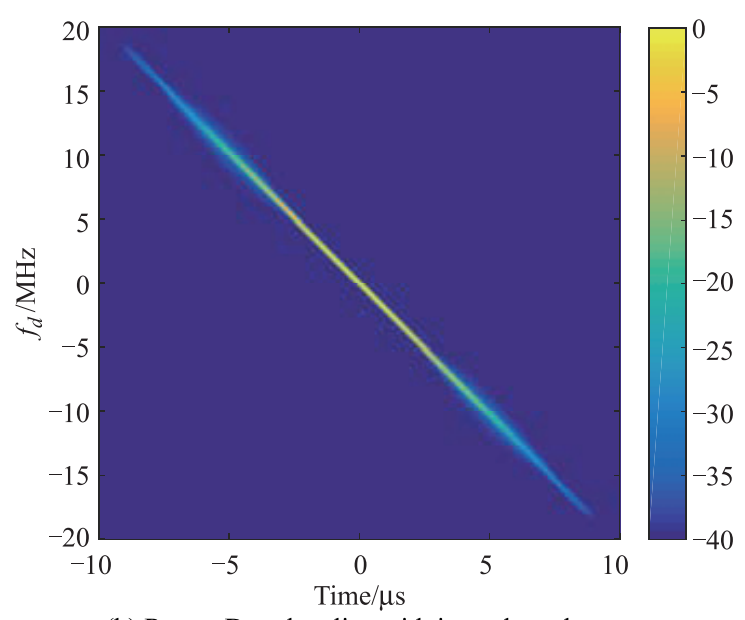

(b) Range-Doppler slice with irregular subarrays

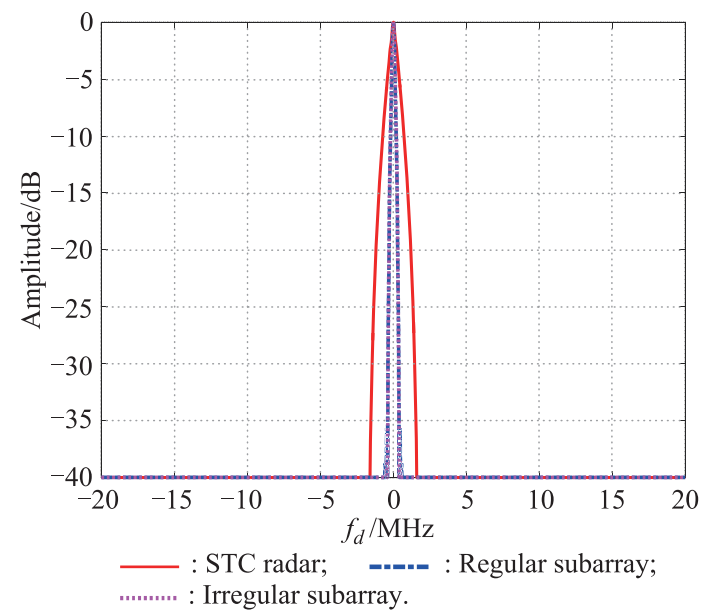

(c) Doppler-dependent plot at $\tau=0$ 


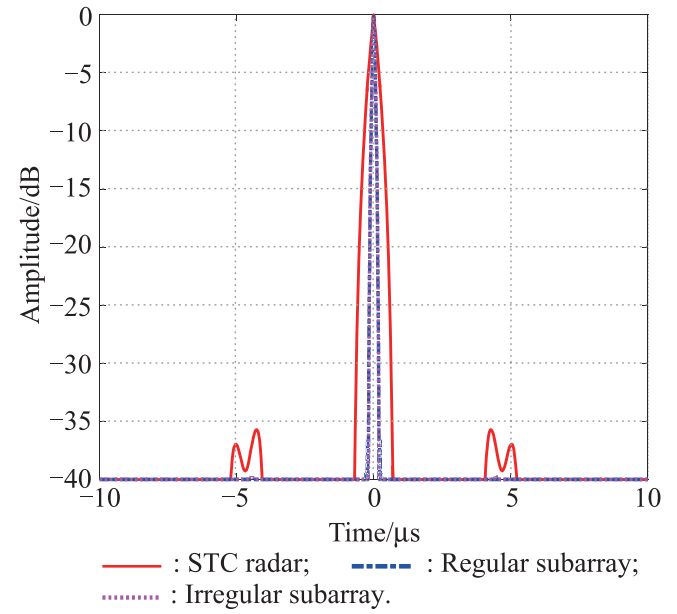

(d) Doppler-dependent plot at $f_{d}=0$

Fig. 6 Range-Doppler 2-D slices of sub-STC radar

(i) Low sidelobes

We notice from Fig. 4 that the subarray-based technique effectively reduces the width of the mainlobe. Besides, sidelobes in the range domain are reduced. Consequently, a "thumbtack-like" 2-D slice in the range-angle domian of the multi-dimensional ambiguity function is achieved in sub-STC radar.

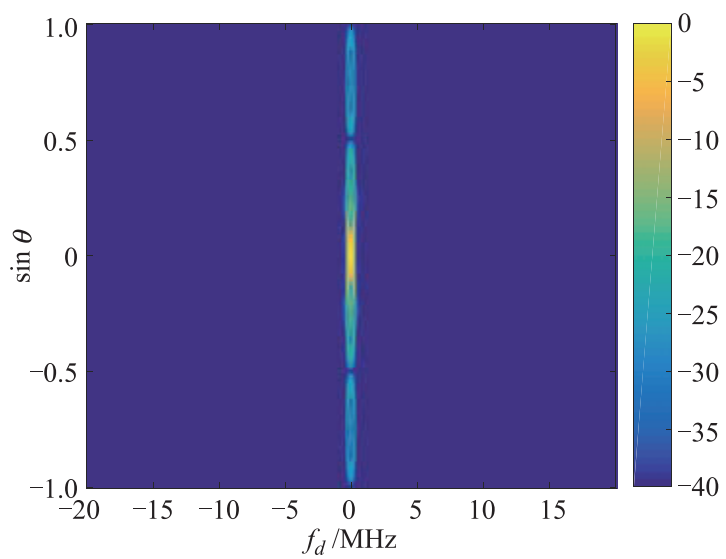

(a) Angle-Doppler 2-D slice regular subarrays

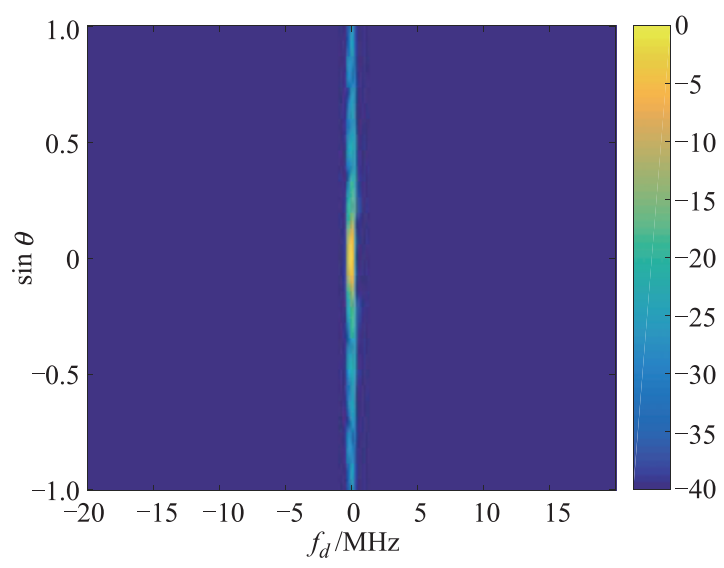

(b) Angle-Doppler 2-D slice regular subarrays

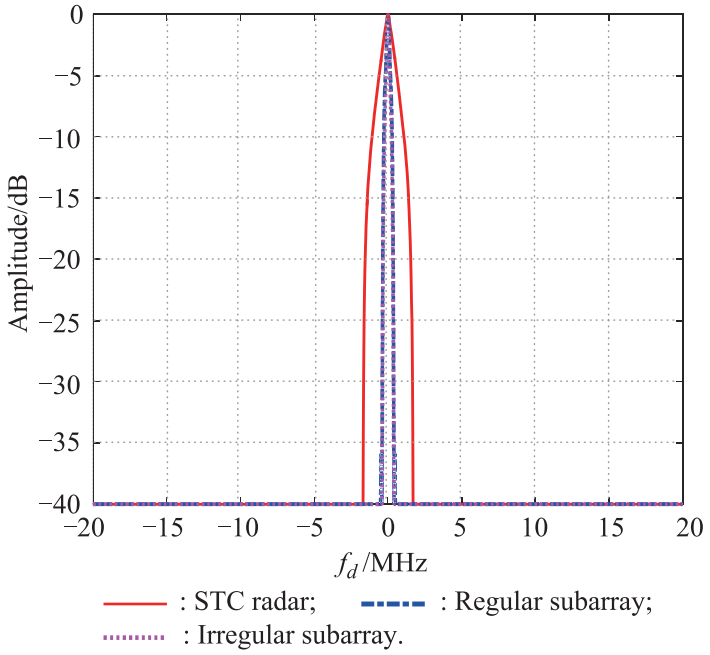

(c) Doppler-dependent plot at $\theta_{0}=0^{\circ}$

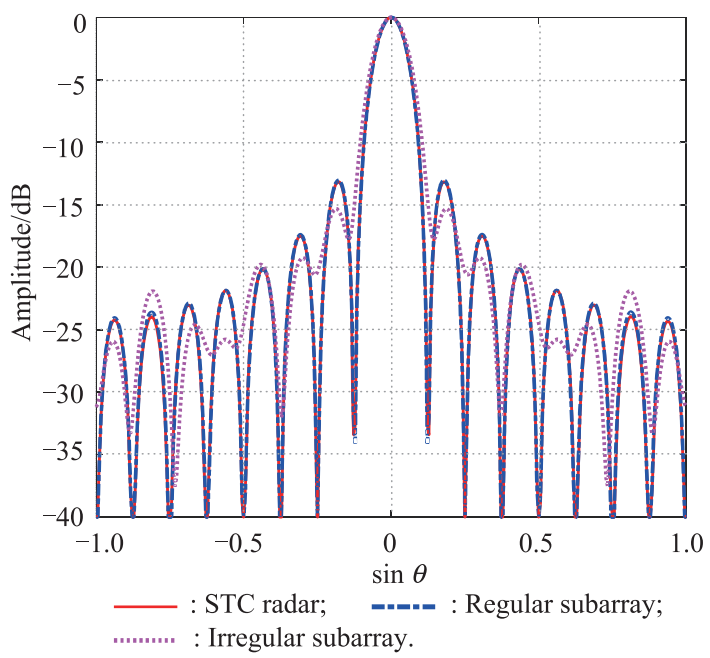

(d) Doppler-dependent plot at $f_{d}=0$

Fig. 7 Angle-Doppler 2-D slices of sub-STC radar

Besides, a satisfactory peak-to-sidelobe ratio is obtained which is reduced to lower than $40 \mathrm{~dB}$.

(ii) Spatial coverage

As shown in Fig. 5, there is a distinct peak at $0^{\circ}$ in the angle-angle 2-D slice of the multi-dimensional ambiguity function. It means that the energy are more focused at $\theta=\theta_{0}=0^{\circ}$. Compared with the STC radar without subarrays, the main diagonal line which demonstrates the uniform angular coverage is corrupted and the improvement of range resolution is at the cost of losing the wide spatial coverage. In the sub-STC radar, the radiated signals are summed coherently at $\theta_{0}=0^{\circ}$ in each subarray, however, the uniform spatial angle coverage degrades.

(iii) Resolution

The improved range resolution and Doppler resolution are shown in Fig. 6. And Fig. 6(c) clearly demonstrates the recovering of the Doppler resolution which is useful 
for detection of slow targets. Fig. 6(d) presents the angledependent plot and it can be seen that the range resolution is improved, which can reach a relatively same result of the conventional phased array radar.

From these figures with different 2-D slices of the multidimensional ambiguity function, the results reveal that the proposed subarray-based method has strengths in improved resolution and reduced sidelobes, but it has spatial coverage loss. The results coincide with our theoretical analyses. Moreover, compared with the STC radar, smaller maximum time delay and summation of time delays are required, which is easier to implement for practical systems.

\section{Conclusions}

In this paper, a subarray-based space-time coding radar, termed as the sub-STC radar is proposed, in which one specific waveform is transmitted but a small time offset is shifted across adjacent subarrays. The multi-dimensional ambiguity function in the sub-STC radar is defined as a function of four parameters including angle, probing direction, range and Doppler. Hence, resolutions not only in the range and Doppler domains but also in the spatial angle domain are considered. The analyses on the properties of the multi-dimensional ambiguity function with regard to the spatial angular coverage, resolution performance and low sidelobes performance are given. It can be found that in the conventional STC radar, if the element number increases, the range resolution is reduced. However, the proposed subarray-based method increases the DOF and improves the resolution in the range domain. Furthermore, the multidimensional ambiguity function with low sidelobes can be achieved. It is concluded that the sub-STC radar characterizes improved resolution, reduced sidelobes, but spatial coverage loss.

\section{References}

[1] TANG B, LI J. Spectrally constrained MIMO radar waveform design based on mutual information. IEEE Trans. on Signal Processing, 2019, 67(3): 821-834.

[2] ZHANG Z Y, ZHANG J Y. Tracking multiple target in MIMO radar via adaptive asymmetric joint diagonalization with deflation. Journal of Systems Engineering and Electronics, 2018, 29(4): $731-741$.

[3] CHENG Z Y, HE Z S, ZHANG S M, et al. Constant modulus waveform design for MIMO radar transmit beampattern. IEEE Trans. on Signal Processing, 2017, 65(18): 4912-4923.

[4] CUI G L, LI H B, RANGASWAMY M. MIMO radar waveform design with constant modulus and similarity constraints. IEEE Trans. on Signal Processing, 2014, 62(2): 343-353.

[5] HE H, STOICA P, LI J. Designing unimodular sequence sets with good correlations - including an application to MIMO radar. IEEE Trans. on Signal Processing, 2009, 57(11): $4391-$ 4405.

[6] AKÇAKAYA M, NEHORAI A. MIMO radar sensitivity analysis for target detection. IEEE Trans. on Signal Processing,
2011, 59(7): $3241-3250$.

[7] DAUM F, HUANG J. MIMO radar: snake oil or good idea. IEEE Trans. on Aerospace Electronic System Magazines, 2009, 24(5): $8-12$.

[8] CHEVALIER F L. Space-time transmission and coding for airborne radars. Radar Science and Technology, 2008, 6(6): 411 421.

[9] BABUR G, AUBRY P, CHEVALIER F L. Space-time codes for active antenna systems: comparative performance analysis. Proc. of the IET International Radar Conference, 2013: 1-6.

[10] FAUCON T, PINAUD G, CHEVALIER F L. Mismatched filtering for circulating space-time codes. Proc. of the IET International Radar Conference, 2015: 1-7.

[11] BABUR G, AUBRY P, CHEVALIER F L. Simple transmit diversity technique for phased array radar. IET Radar Sonar \& Navigation, 2016, 10(6): 1046-1056.

[12] ROUSSEL K, BABUR G, CHEVALIER F L. Optimization of low sidelobes radar waveforms: circulating codes. Proc. of the International Radar Conference, 2014: $1-6$.

[13] BABUR G, AUBRY P, CHEVALIER F L. Antenna coupling effects for space-time radar waveforms: analysis and calibration. IEEE Trans. on Antennas and Propagation, 2014, 62(5): $2572-2586$.

[14] WOODWARD P M. Probability and information theory with applications to radar. Whitstable, UK: McGraw-Hill, 1953.

[15] MBROOKNER E. Multidimensional ambiguity function of linear, interferometer, antenna arrays. IEEE Trans. on Antennas and Propagation, 1964, 12(5): 551-561.

[16] BHATTA A, MISHRA A K. Ambiguity function and CramerRao lower bound calculation for CommSense system. IEEE Trans. on Aerospace and Electronic Systems, 2018, 54(4): 2067-2074.

[17] LI H, QIN Y L, LI Y P, et al. Parameter estimation of intrapulse modulation for poly-phase coded pulse based on AF. Systems Engineering and Electronics, 2010, 32(9): $1823-$ 1827. (in Chinese)

[18] ANTONIO G S, FUHRMANN D R, ROBEY F C. MIMO radar ambiguity functions. IEEE Journal of Selected Topics in Signal Processing, 2007, 1(1): $167-177$.

[19] YANG M L, CHEN B X, QI F L, et al. Ambiguity function of multiple-carrier frequency MIMO radar. Systems Engineering and Electronics, 2009, 31(1): 5-8. (in Chinese)

[20] KHAN W, QURESHI I M, SULTAN K. Ambiguity function of phased-MIMO radar with colocated antennas and its properties. IEEE Trans. on Geoscience and Remote Sensing Letters, 2014, 11(7): 1220-1224.

[21] WANG W Q, DAI M, ZHENG Z. FDA radar ambiguity function characteristics analysis and optimization. IEEE Trans. on Aerospace and Electronic Systems, 2018, 54(3): 1368 - 1380.

[22] DESODT G, MICHEL G E, GUYVARCH J P, et al. Multibursts space-time coding for active antenna systems. Proc. of the IEEE International Radar Conference, 2014: 1-6.

[23] WEN C, TAO M L, PENG J Y, et al. Clutter suppression for airborne FDA-MIMO radar using multi-waveform adaptive processing and auxiliary channel STAP. Signal Processing, 2019, 154: 280-293.

[24] WANG W Q, DAI M M, ZHENG Z. FDA radar ambiguity function characteristics analysis and optimization multiwaveform adaptive processing and auxiliary channel STAP. Signal Processing, 2019, 154: 280-293.

[25] WANG W Q. Overview of frequency diverse array in radar and navigation applications. IET Radar, Sonar \& Navigation, 2016, 10(6): $1001-1012$.

[26] HARRIS F J. On the use of windows for harmonic analysis with the discrete Fourier transform. Proceedings of the IEEE, 
1978, 66(1): $172-204$.

[27] ZENG G Q, LI S Y, ZHANG Y, et al. Low side lobe pattern synthesis using projection method with genetic algorithm for truncated cone conformal phased arrays. Journal of Systems Engineering and Electronics, 2014, 25(4): 554-559.

[28] OMAR O K, DEBBAT F, STAMBOULI A B. Null steering beamforming using hybrid algorithm based on honey bees mating optimization and tabu search in adaptive antenna array. Progress in Electromagnetics Research C, 2012, 32: 65-80.

[29] BEVELACQUA P J, BALANIS C A. Geometry and weight optimization for minimizing sidelobes in wideband planar arrays. IEEE Trans. on Antennas and Propagation, 2009, 57(4): $1285-1289$.

\section{Biographies}

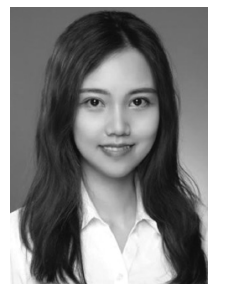

LAN Lan was born in 1993. She received her B.S. degree in electronic engineering from Xidian University, Xi'an, China, in 2015. She is currently working toward her Ph.D. degree in the National Laboratory of Radar Signal Processing, Xidian University. Her research interests include frequency diverse array radar systems, MIMO radar signal processing and electronic counter-countermeasures. E-mail: lanlan_xidian@foxmail.com

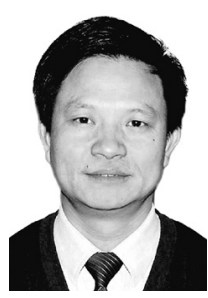

LIAO Guisheng was born in 1963. He received his B.S. degree from Guangxi University, Guangxi, China, and M.S. and Ph.D. degrees from Xidian University, Xi' an, China, in 1985, 1990, and 1992, respectively. $\mathrm{He}$ is currently a professor at the $\mathrm{Na}$ tional Laboratory of Radar Signal Processing, Xidian University. He has been a senior visiting scholar in the Chinese University of Hong Kong,
Hong Kong. His research interests include array signal processing, spacetime adaptive processing, synthetic aperture radar (SAR) ground moving target indication, and distributed small satellite SAR system design. He is a member of the National Outstanding Person and the Cheung Kong Scholars in China.

E-mail: liaogs@xidian.edu.cn

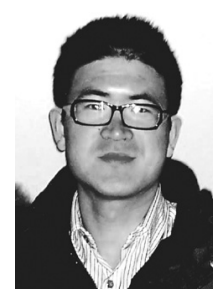

XU Jingwei was born in 1987. He received his B.S. degree in electronic and information engineering, and $\mathrm{Ph} . \mathrm{D}$. degree in signal and information processing, both from Xidian University, China, in 2010 and 2015, respectively. He is currently an associate professor in the National Laboratory of Radar Signal Processing, Xidian University. His research interests include robust adaptive beamforming, frequency diverse array radar systems, space-time adaptive processing (STAP)-based radar signal processing, and MIMO radar signal processing.

E-mail: xujingwei1987@163.com

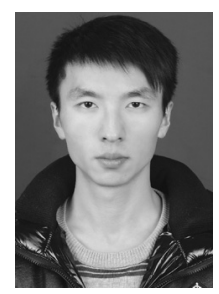

WANG Hanbing was born in 1990. He received his B.S. degree in detection guidance and control technology from Xidian University, Xi'an, China, in 2013, and M.S. degree in circuits and systems from Northwestern Polytechnical University, Xi' an, China, in 2016. He is currently working toward his Ph.D. degree in the National Laboratory of Radar Signal Processing, Xidian University. His research interests include waveform diversity and array radar signal processing. E-mail: hbwang_10@163.com 